12,09

\title{
Влияние резонансного ИК-лазерного излучения на магнетопоглощение в квантовых проволоках
}

\author{
(C) Э.П. Синявский ${ }^{1}$, С.А. Карапетян ${ }^{2}$, Н.С. Костюкевич ${ }^{2,9}$ \\ ${ }^{1}$ Институт прикладной физики АН Молдовы, \\ Кишинев, Молдова \\ ${ }^{2}$ Приднестровский государственный университет им. Т.Г. Шевченко, \\ Тирасполь, Молдова \\ ฯ E-mail: gravitonchik@gmail.com
}

(Поступила в Редакцию 25 февраля 2016 г.

В окончательной редакции 6 сентября 2016 г.)

\begin{abstract}
Вычислен коэффициент межзонного поглощения слабой электромагнитной волны в квантовых проволоках в поперечном магнитном поле и в поле интенсивного лазерного излучения. Показано, что когда частота лазерного излучения равна или частоте размерного квантования (размерно-инфракрасный резонанс) или гибридной частоте (магнито-инфракрасный резонанс), то лазерная подсветка может определять форму осцилляций поглощения. В частности, показано, что второй пик магнетопоглощения расщепляется на два пика, полуширина и расстояние между которыми зависят от интенсивности резонансного лазерного излучения. Обсуждается влияние поляризации ИК-излучения на межзонное поглощение в квантовых проволоках. Исследована динамика частотной зависимости коэффициента поглощения света при увеличении интенсивности резонансного лазерного излучения.
\end{abstract}

Авторы приносят благодарность STCU (грант 5929) за частичную финансовую поддержку работы.

DOI: 10.21883/FTT.2017.04.44280.066

В настоящее время 0-мерные и 1-мерные системы привлекают внимание исследователей благодаря своим необычным оптическим и оптоэлектронным свойствам [1], которые могут быть использованы для разработки новых оптоэлектронных приборов [2,3]. Создание мощных источников когерентного оптического излучения дало мощный импульс к исследованию и поискам новых нелинейных эффектов в физике твердого тела и явилось эффективным инструментом для изучения оптических свойств конденсированных сред. Особое место при исследовании оптических свойств занимает случай, когда частота излучения попадает в резонанс с какой-либо частотой квантовой системы. Примером является задача о двойном оптическом резонансе [4] (резкое изменение коэффициента поглощения света при переходе электрона из основного электронного состояния системы в возбужденное в присутствии интенсивного излучения с частотой резонансной смежному переходу). Исследование резонансных эффектов дает возможность получить детальную информацию о микроскопических свойствах твердого тела, исследовать (и это очень важно) нелинейные эффекты при небольших интенсивностях лазерной подсветки. Например, интересные особенности в межзонном магнетопоглощении света возникают в случае, когда полупроводниковый материал облучается интенсивной электромагнитной волной с частотой $\omega_{L}$ равной циклотронной частоте [5-7] (магнетоинфракрасный резонанс - МИКР). В настоящей работе рассматриваются оптические свойства квантовых проволок в поле резонансного лазерного излучения. Эти исследования являются интересными так как даже в отсутствии магнитного поля в нанопроволоке на дне каждой размерно-квантовой зоны проводимости возникают особенности плотности электронных состояний. Это обстоятельство приводит к более заметному влиянию резонансного лазерного излучения $\left(\omega_{L}\right.$ равно частоте размерного квантования) на межзонные оптические переходы, чем это происходит в квантовых ямах [6,7].

Рассмотрим квантовую проволоку в однородном магнитом поле $\mathbf{B}$, направленном перпендикулярно оси исследуемой наноструктуры. Энергетический спектр электрона в параболической нанопроволоке известен [8]

$$
\begin{gathered}
E_{a}=\frac{\hbar^{2} k_{x}^{2}}{2 m_{e}^{*}}+\hbar \Omega_{e}\left(n+\frac{1}{2}\right)+\hbar \omega_{e}\left(m+\frac{1}{2}\right), \\
m_{e}^{*}=m_{e}\left(\frac{\Omega_{e}}{\omega_{e}}\right)^{2}, \quad \Omega_{e}=\left[\omega_{e}^{2}+\omega_{c}^{2}\right]^{1 / 2}, \quad \omega_{c}=\frac{e B}{m_{e} c},
\end{gathered}
$$

где $\hbar \omega_{e}-$ энергия размерного квантования электрона массы $m_{e}$ в зоне проводимости, $\omega_{c}-$ циклотронная частота. Аналогично можно записать для электрона в валентной зоне.

В дальнейшем исследуем оптические свойства полупроводниковых квантовых проволок (КП), схема энергетических зон которых изображена на рис. 1. $E_{g}^{*}-$ ширина запрещенной зоны КП в магнитном поле, $\omega_{v}$, $\Omega_{v}$ - соответственно частота размерного квантования и гибридная частота носителей в валентной зоне.

В работе вычисляется коэффициент межзонного поглощения слабой электромагнитной волны в поле лазерного излучения, частота которого $\omega_{L}$ равна $\omega_{e}$ (размерно-инфракрасный резонанс) или $\Omega_{e}$ (магнитноинфракрасный резонанс). Так как для типичных полу- 


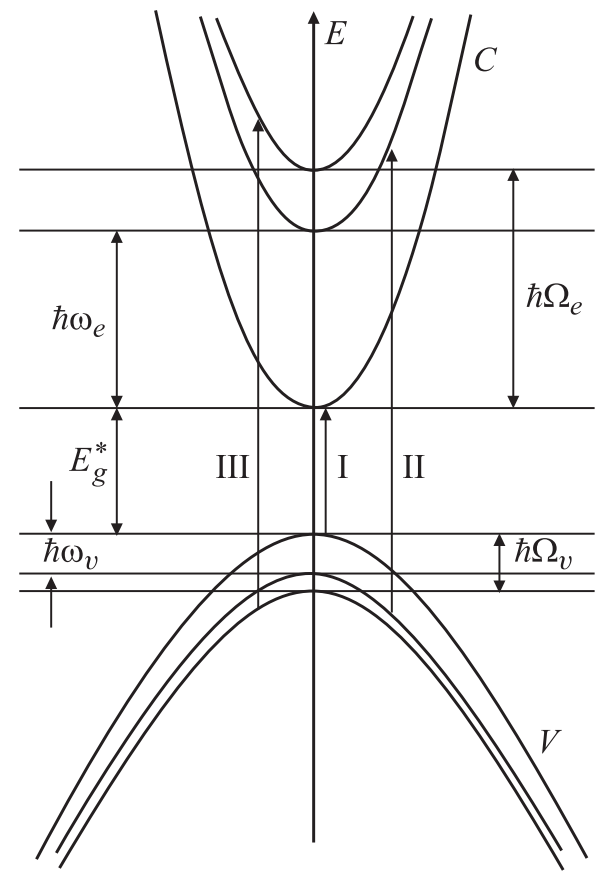

Рис. 1. Схема энергетических зон полупроводниковой квантовой проволоки в поперечном магнитном поле и оптические переходы, рассматриваемые в работе.

проводниковых наноструктур эффективная масса электронов в зоне проводимости значительно меньше массы дырок $\left(m_{e} \ll m_{v}\right)$, то взаимодействием интенсивного ИК-излучения с электронами в валентной зоне в дальнейшем пренебрегаем $\left(\omega_{e} \gg \omega_{v}\right)$.

Гамильтониан в представлении вторичного квантования для электронов в зоне проводимости в состоянии $\alpha$ в поле одномодового лазерного излучения поляризацией $\xi$ записывается в следующем виде

$$
\begin{aligned}
\hat{H}= & \sum_{\alpha} \varepsilon_{\alpha} a_{\alpha}^{+} a_{\alpha}+\hbar \omega_{L} b^{+} b \\
& +\left[\frac{2 \pi \hbar e^{2}}{V \omega_{L}}\right]^{\frac{1}{2}} \sum_{\alpha \alpha_{1}}\left|\frac{P_{\alpha \alpha_{1}} \xi}{m_{e}}\right|^{2} a_{\alpha}^{+} a_{\alpha_{1}}\left(b^{+}+b\right) .
\end{aligned}
$$

Здесь $a_{\alpha}^{+}\left(a_{\alpha}\right), b^{+}(b)$ - операторы рождения (уничтожения) электронов в состоянии $\alpha$ и фотонов.

Расчет матричных элементов оператора импульса $\mathbf{P}_{\alpha \alpha_{1}}$ на волновых функциях квантовой параболической проволоки в продольном магнитном поле [1] не представляет труда. В результате

$$
\begin{gathered}
\left|\mathbf{P}_{\alpha \beta}^{Y}\right|^{2}=\frac{\hbar \omega_{e}\left(1+\delta^{2}\right)^{1 / 2} m_{e}}{2} \delta_{k_{x}, k_{x}^{\prime}} \delta_{m, m_{1}} \\
\times\left\{n \delta_{n, n_{1}+1}+(n+1) \delta_{n, n_{1}-1}\right\} \\
\left|\mathbf{P}_{\alpha \beta}^{X}\right|^{2}=\frac{\delta^{2}}{\left(1+\delta^{2}\right)^{1 / 2}}\left|\mathbf{P}_{\alpha \beta}^{Y}\right|^{2}, \quad \delta=\left(\frac{\omega_{c}}{\omega_{e}}\right) \\
\left|\mathbf{P}_{\alpha \beta}^{Z}\right|^{2}=\frac{\hbar \omega_{e} m_{e}}{2} \delta_{k_{x}, k_{x}^{\prime}} \delta_{n, n_{1}}\left\{m \delta_{m, m_{1}+1}+(m+1) \delta_{m, m_{1}-1}\right\}
\end{gathered}
$$

Из выражения (2) следует, что линейно $X$-поляризованная волна лазерного излучения (электромагнитная волна распространяется перпендикулярно оси нанопроволоки) в отсутствии магнитного поля $(\delta=0)$ не взаимодействует с зонными носителями. Согласно (2), линейно $Y$-поляризованная волна (лазерное излучение распространяется вдоль оси квантовой проволоки) смешивает гибридные состояния $\left(\hbar \omega_{L}=\hbar \Omega_{e}\right)$, а $Z$-поляризованная волна (напряженность электрического поля лазерного излучения $\mathbf{E} \perp \mathbf{B})$ смешивает только размерноквантованные состояния $\left(\hbar \omega_{L}=\hbar \omega_{e}\right)$.

Расчет коэффициента межзонного поглощения слабой электромагнитной волны частоты $\Omega$ в поле резонансного лазерного излучения для квантовых проволок производился с использованием формулы Кубо [9] и методики, развитой в [5]. В результате в резонансном случае $\omega_{L}=\Omega_{e}$ для стабильно генерирующего ИК-лазерного излучения $Y$-поляризации получаем

$$
\begin{gathered}
K(\Omega)=K_{0} \sum_{n m}\left|\left\langle\alpha_{c} \mid \alpha_{v}\right\rangle\right|^{2} \\
\times \int_{-\infty}^{\infty} d k_{x} \int_{-\infty}^{\infty} d t e^{-a t^{2}} e^{\frac{\gamma_{0}|t|}{\left|k_{x}\right|}} L_{n}\left(2 a t^{2}\right) \exp \left\{\frac { i t } { \hbar } \left[\hbar \Omega-E_{g}^{*}\right.\right. \\
\left.\left.-\frac{\hbar^{2} k_{x}^{2}}{2 \mu^{*}}-m\left(\hbar \omega_{e}+\hbar \omega_{v}\right)-n\left(\hbar \Omega_{e}+\hbar \Omega_{v}\right)\right]\right\}, \\
K_{0}=\frac{e^{2}}{n_{0} c S \hbar \Omega}\left|\frac{\mathbf{P}_{C V} e}{m_{0}}\right|^{2}, \quad a=\frac{e^{2} E^{2}}{8 m_{e} \hbar \Omega_{e}}, \\
\frac{1}{\mu^{*}}=\frac{1}{m_{e}^{*}}+\frac{1}{m_{v}^{*}}, \quad m_{v}^{*}=m_{v}\left(\frac{\Omega_{v}}{\omega_{v}}\right)^{2},
\end{gathered}
$$

где $\left\langle\alpha_{c} \mid \alpha_{v}\right\rangle$ - матричный элемент сглаженных волновых функций электрона в зоне проводимости и в валентной зоне, $L_{n}(z)$ - полиномы Лаггера, $E-$ напряженность электрического поля лазерного излучения, $S-$ сечение квантовой проволоки.

При записи (3) учитывалось взаимодействие носителей с шероховатой поверхностью или с длинноволновыми акустическими фононами в приближении времени релаксации [10]. $\gamma_{0} /\left|k_{x}\right|$ - описывает вероятность рассеяния электронов в единицу времени или на шероховатой поверхности [11] или упругое рассеяние на акустических фононах [12].

Запишем коэффициент поглощения света, связанный с переходом электрона из нижайшей валентной зоны $(m=n=0)$ в нижайшую размерно-квантованную зону проводимости $(n=m=0)$. (Оптический переход $I$ на рис. 1). Интегрирование по $t$ в (3) проводится точно, в результате, после замены

$$
\frac{\hbar^{2} k_{x}^{2}}{2 \mu^{*}}=\hbar \omega_{f} x^{2}\left(\omega_{f}^{3}=\frac{\hbar \gamma_{0}^{2}}{2 \mu^{*}}\right)
$$


соотношение (3) записывается в виде $\left(L_{0}(z)=1\right)$

$$
\begin{aligned}
& K(\Omega)= K_{0} \sum_{n m}\left|\left\langle\alpha_{c} \mid \alpha_{v}\right\rangle\right|^{2} 2\left[\frac{2 \pi \mu^{*} \omega_{f}}{\hbar a}\right]^{\frac{1}{2}} \\
& \times \operatorname{Re} \int_{0}^{\infty} d x e^{f^{2}(x)}[1-\Phi(f(x))], \\
& \Phi(z)=\frac{2}{\sqrt{\pi}} \int_{0}^{z} e^{-\tau^{2}} d \tau,
\end{aligned}
$$

$f(x)=\left(\frac{\omega_{f}^{2}}{4 a}\right)^{\frac{1}{2}} \frac{1}{x}\left[1-i x\left(\frac{\Delta}{\hbar \omega_{f}}-x^{2}\right)\right], \quad \Delta=\hbar \Omega-E_{g}^{*}$.

В отсутствие лазерного излучения $a=0$ из (4) при $\omega_{f}^{2} \gg 1$ можно получить выражение для коэффициента межзонного поглощения света в нанопроволоках в однородном магнитном поле [13]. Если же $\omega_{f}^{2} / 4 a \ll 1$, то форма линии межзонного поглощения света (высота, полуширина) полностью определяется интенсивностью лазерного излучения, и коэффициент поглощения света согласно (4) записывается следующим образом:

$$
K(\Omega)=K_{0}\left|\left\langle\alpha_{c} \mid \alpha_{v}\right\rangle\right|^{2} 2\left[\frac{2 \pi \mu^{*}}{\hbar^{2} a}\right]^{\frac{1}{2}} e^{-\frac{\Delta^{2}}{4 a \hbar^{2}}} \int_{0}^{\infty} d x e^{-\frac{x^{4}}{4 a \hbar^{2}}+\frac{\Delta x^{2}}{2 a \hbar^{2}}} .
$$

После интегрирования по $x$ выражение (5) примет вид

$$
\begin{aligned}
K(\Omega) & =K_{0}\left|\left\langle\alpha_{c} \mid \alpha_{v}\right\rangle\right|^{2}\left[\frac{\pi \mu^{*}|\Delta|}{\hbar^{2} a}\right]^{\frac{1}{2}} \\
& \times \begin{cases}e^{-z} K_{1 / 4}(z), & \Delta \leq 0 \\
\frac{\pi}{\sqrt{2}} e^{-z}\left\{I_{1 / 4}(z)+I_{-1 / 4}(z)\right\}, & \Delta>0\end{cases}
\end{aligned}
$$

где $K_{v}(z)-$ функция Макдональда, $I_{v}(z)-$ модифицированная функция Бесселя, $z=\Delta^{2} / 8 a \hbar^{2}$.

На рис. 2 представлена частотная зависимость коэффициента поглощения света (в относительных единицах) при различных значениях интенсивности лазерного излучения. Кривые 1, 2, 3 получены соответственно при $\xi=0.25,0.05,0.01\left(\xi=\omega_{f}^{2} / 4 a\right)$. Как следует из рис. 2 с ростом интенсивности ИК-излучения ( $\xi$ уменьшается при фиксированном значении $\omega_{f}$ ) форма линии поглощения изменяется: величина максимума поглощения уменьшается, а полуширина увеличивается. Заметим, что уже при $\xi \leq 1$ коэффициент межзонного поглощения света полностью определяется интенсивностью ИК-лазерного излучения.

Рассмотрим межзонное поглощение света в области второго пика магнетопоглощения (оптический переход II на рис. 1) при $\omega_{L}=\Omega_{e}$ (магнитоинфракрасный резонанс). В этом случае коэффициент поглощения света согласно (3) при $m=0, n=1\left(L_{1}\left(2 a t^{2}\right)=1-2 a t^{2}\right)$

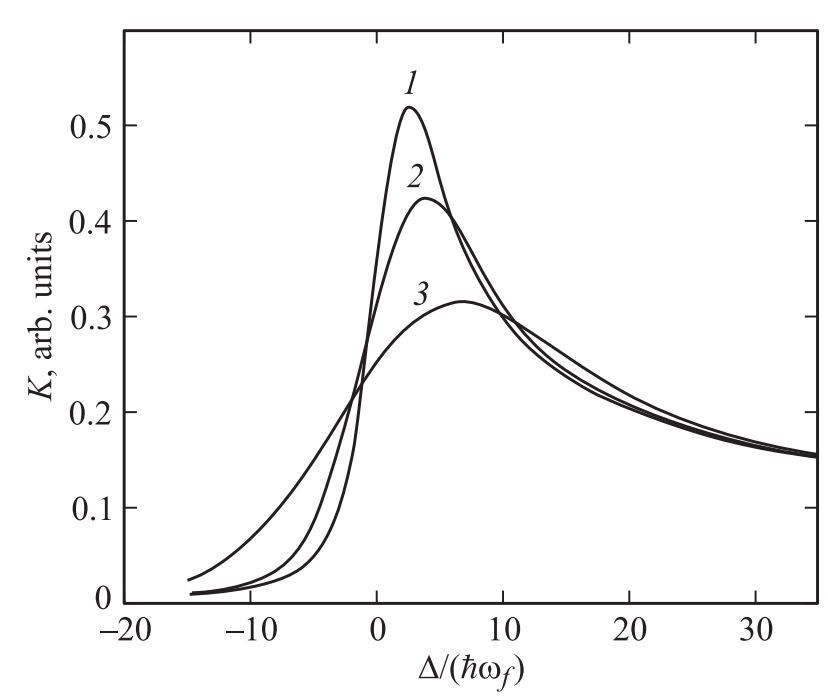

Pис. 2. Зависимость первого пика межзонного поглощения света (в относительных единицах) от $\Delta / \hbar \omega_{f}$. Кривые $1,2,3$ вычислены $\xi=0.25,0.05,0.01$ соответсвенно.

принимает следующий вид

$$
\begin{aligned}
& K(\Omega)=K_{0}\left|\left\langle\tilde{\alpha}_{c} \mid \tilde{\alpha}_{v}\right\rangle\right|^{2} 4\left[\frac{2 \mu^{*} \omega_{f}}{\hbar a}\right]^{\frac{1}{2}} \\
& \quad \times \operatorname{Re} \int_{0}^{\infty} d x f(x)\left\{-\sqrt{\pi} f(x) e^{f^{2}(x)}[1-\Phi(f(x))]+1\right\} .
\end{aligned}
$$

При $\xi=\omega_{f}^{2} / 4 a \gg 1$ (лазерное излучение отсутствует) из (7) следует выражение для коэффициента поглощения слабой электромагнитной волны, полученное в [7]. При $\xi<1$ (форма линии поглощения определяется интенсивностью резонансного ИК-излучения) получено

$$
\begin{aligned}
& K(\Omega)= K_{0}\left|\left\langle\tilde{\alpha}_{c} \mid \tilde{\alpha}_{v}\right\rangle\right|^{2} \pi z\left[\frac{2 \mu^{*} \pi}{\hbar}\left(\frac{8 z}{a}\right)^{1 / 2}\right]^{1 / 2} \\
& \times e^{-z}\left\{-\left(I_{3 / 4}(z)+\operatorname{sign} \Delta I_{-3 / 4}(z)\right)\right. \\
&\left.+\left(1+\frac{1}{4 z}\right)\left(I_{-1 / 4}(z)+\operatorname{sign} \Delta I_{1 / 4}(z)\right)\right\}, \\
& \operatorname{sign} \Delta= \begin{cases}1, & \Delta>0, \\
-1, & \Delta<0,\end{cases}
\end{aligned}
$$

где $\left\langle\tilde{\alpha}_{c} \mid \tilde{\alpha}_{v}\right\rangle-$ матричный элемент на сглаженных волновых функциях носителей в возбужденном состоянии валентной зоны $(m=0, n=1)$ и в возбужденном состоянии размерно-квантованной зоны проводимости.

На рис. 3 приведена частотная зависимость второго пика магнетопоглощения при различных значениях $\xi$. Кривые 1, 2, 3 вычислены при $\xi=0.25,0.05,0.01$ соответственно. Как следует из рис. 3 , с ростом напряженности $E$ электрического поля пик магнетопоглощения (1) 


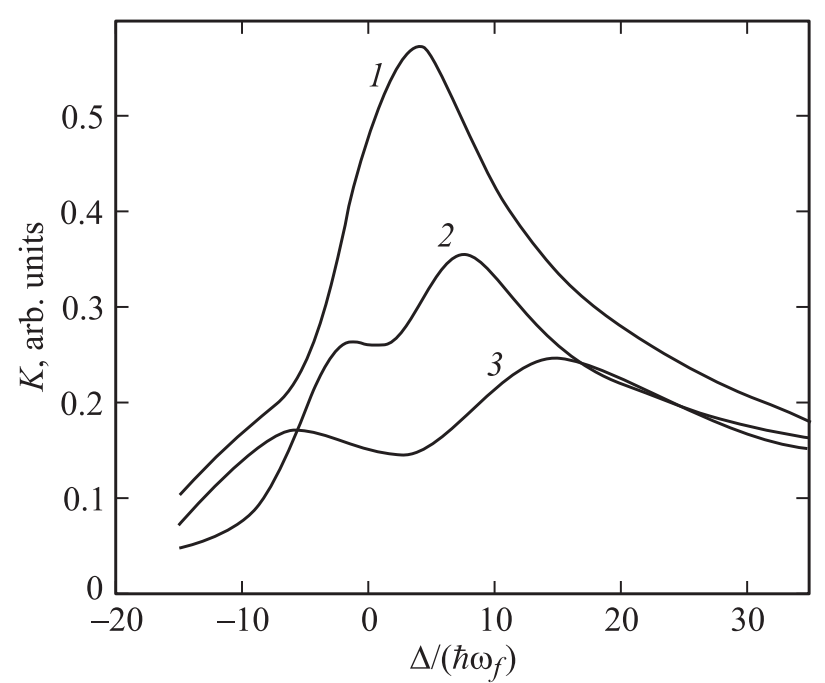

Рис. 3. Зависимость второго пика магнетопоглощения (в относительных единицах) от $\Delta / \hbar \omega_{f}$ при различных значениях интенсивности резонансного $\left(\omega_{L}=\Omega_{e}\right)$ лазерного излучения. Кривые 1,2, 3 вычислены $\xi=0.25,0.05,0.01$ соответсвенно.

деформируется и при $\xi \ll 1$ расщепляется на два пика. При этом расстояние между ними и их полуширина увеличивается. Расщепление второго пика поглощения связано с тем, что при $\omega_{L}=\Omega_{e}$ возбужденное гибридное состояние $(n=1)$ двукратно вырождено, и при взаимодействии с ИК-лазерным излучением оно расщепляется. Эта ситуация близка к двойному оптическому резонансу (ДОР) на межзонных переходах в объемных материалах [4].

Заметим, что $n$-пик магнетопоглощения расщепляется на $n$ пиков. Если рассматривать случай $z$-поляризованной электромагнитной волны лазерного излучения, когда $\omega_{L}=\omega_{e}$ (размерно-инфракрасный резонанс), то частотная зависимость коэффициента межзонного поглощения света (оптический переход III на рис. 1) качественно не отличается от частотной зависимости, приведенной на рис. 2 и на рис. 3.

Пусть при некотором значении напряженности электрического поля $E_{c}$ интенсивной электромагнитной волны вклад лазерного излучения в полуширину магнетоосцилляций примерно такой же, как вклад, определяемый рассеянием носителей на шероховатой поверхности $(\xi=1)$. Естественно при $E_{c}<E$ форма линии межзонного поглощения слабой электромагнитной волны полностью определяется внешней лазерной подсветкой. Для типичных параметров полупроводниковой нанопроволоки $m_{e}=0.06 m_{0}, m_{v}=0.4 m_{0}, \sqrt[3]{\gamma_{0}}=20 \AA$ (такое значение $\sqrt[3]{\gamma_{0}}$ хорошо описывает большие значения подвижности $\mu \propto 10^{4} \mathrm{~cm}^{2} / \mathrm{V} \cdot \mathrm{s}$, характерные для квантовых проволок) при $R_{0}=10^{3} \AA, E_{c}=7 \mathrm{~V} / \mathrm{cm}$ для лазера $\mathrm{H}_{2} \mathrm{O}$ $\left(\hbar \omega_{L}=0.044 \mathrm{eV}\right)$. Следовательно, резонансное лазерное излучение заметно влияет на частотную зависимость межзонного поглощения света при небольших, вполне экспериментально доступных значениях интенсивности
ИК-лазерного излучения. Последнее обстоятельство является важным, поскольку возникает уникальная возможность управления кинетическими эффектами в поле резонансного лазерного излучения, что может найти практическое применение.

\section{Список литературы}

[1] R. Agarwal, C.M. Lieber. Appl. Phys. A 85, 209 (2006).

[2] Linyou Cao, J.S. White, Joon-Shik Park, J.A. Schuller, B.M. Clemens, M.L. Brongersma. Nature Mater. 8, 643 (2009).

[3] A.B. Greytak, C.J. Barrelet, Yat Li, Charlis M. Lieber. Appl. Phys. Lett. 87, 151103 (2005).

[4] Е.Ю. Перлин, В.А. Каварский. ФТТ 12, 31056 (1970).

[5] Э.П. Синявский. ФТТ 16, 3201 (1974).

[6] Э.П. Синявский, С.А. Карапетян. ФТТ 48, 906 (2006).

[7] Э.П. Синявский, Е.И. Брусенская. ФТТ 44, 1116 (2002).

[8] F.M. Hashimzade, T.G. Ismailov, B.H. Hehdiev, S.T. Pavlov. Phys. Rev. B 71, 165331 (2005).

[9] R. Kubo. J. Phys. Soc. Jpn. 12, 570 (1957).

[10] Э.П. Синявский, Р.А. Хамидуллин. ФТП 36, 989 (2002).

[11] Э.П. Синявский, С.А. Карапетян. ФТП 46, 1032 (2012).

[12] Э.П. Синявский, Р.А. Хамидуллин. ФТП 40, 11, 1368 (2006).

[13] Э.П. Синявский, Е.Ю. Канаровский, Н.С. Костюкевич. Оптика и спектроскопия 119, 5, 783 (2015). 